\title{
The alignment approach to nasotracheal intubation
}

\author{
Carrie Goodine, MD • Kathryn Sparrow, MD • Mathieu Asselin, MD • \\ David Hung, BA $\cdot$ Orlando Hung, MD
}

Received: 7 February 2016/Revised: 10 March 2016/Accepted: 24 March 2016/Published online: 4 April 2016

(C) Canadian Anesthesiologists' Society 2016

\section{To the Editor,}

During nasotracheal intubation under direct laryngoscopy (DL), the tip of the Macintosh laryngoscope is advanced into the vallecula, indirectly elevating the epiglottis by applying pressure on the hyoepiglottic ligament. Although this maneuver allows optimal visualization of the glottis, it lifts the larynx away from the tip of the advancing nasotracheal tube (NTT), which generally lies along the posterior pharyngeal wall (Figure, panel A). Most clinicians use Magill forceps to direct the tip of the NTT anteriorly to enter the glottis. This commonly employed technique has limitations. The use of Magill forceps may lead to tearing the NTT cuff. ${ }^{1}$ In addition, advancement of the NTT past the vocal cords may be difficult as the now anteriorly directed NTT often abuts, and is held up on, the anterior tracheal wall (Figure, panel B).

We suggest a conceptual change in the practice of nasotracheal intubation. We suggest maintaining the glottic opening, wherein NTT alignment is the goal of the airway maneuvers instead of obtaining the "optimal view." This method would remove the need to use Magill forceps for successful nasotracheal intubation.

This alignment approach involves two components. The first is to minimize lifting the glottis during laryngoscopy. The conventional teaching for both DL and indirect

Electronic supplementary material The online version of this article (doi:10.1007/s12630-016-0641-y) contains supplementary material, which is available to authorized users.

C. Goodine, MD · K. Sparrow, MD · M. Asselin, MD .

D. Hung, BA · O. Hung, MD ( $₫)$

Department of Anesthesia, Victoria General Hospital, Dalhousie

University, Halifax, NS, Canada

e-mail: hungorla@dal.ca laryngoscopy using a video laryngoscope is to obtain an "optimal glottic view," or grade 1 Cormack-Lehane view (Figure, panel A). This degree of anterior lift creates misalignment of the glottic opening and the NTT (Figure, panel B). We advocate minimal lifting of the glottis by intentionally limiting laryngoscopy to a grade 2 view (Figure, panel C). This principle would probably work best with video laryngoscopy as the primary method for nasotracheal intubation, rather than DL, as suggested by Jones $e t a l .^{2}$ The view obtained on the screen of the video laryngoscope is at least the same as - but is most likely to be one grade better than - that with DL. ${ }^{3}$ A grade 2 view on the screen would provide optimized glottic opening-NTT alignment for nasotracheal intubation, wherein the tip of the NTT is more likely to be directed toward the glottis than toward the esophagus.

The second component of the alignment approach involves the cuff inflation technique. Despite utilizing the "limited" glottic view technique during video laryngoscopy, the tip of the NTT may remain posterior to the glottis (Figure, panel C). Then, instead of using the Magill forceps to pick up and guide the tip of the NTT into the glottis, the cuff of the NTT can be slowly inflated to lift the NTT away from the posterior pharyngeal wall. The cuff inflation technique has been well described as an aid during blind nasotracheal intubation ${ }^{4}$ and with a variety of airway adjuncts. ${ }^{5}$ Cuff inflation should be sufficient to facilitate tracheal intubation while maintaining glottic opening-NTT alignment. Once the tip of the NTT is seen on the video laryngoscope screen, the NTT is advanced to the glottic opening. Slow inflation of the cuff with $10-20 \mathrm{~mL}$ of air elevates the NTT tip and aligns it with the glottic opening. The NTT is advanced until the tip has passed through the vocal cords. The cuff is then deflated to allow the NTT to advance through the glottis (Figure, panel D). 

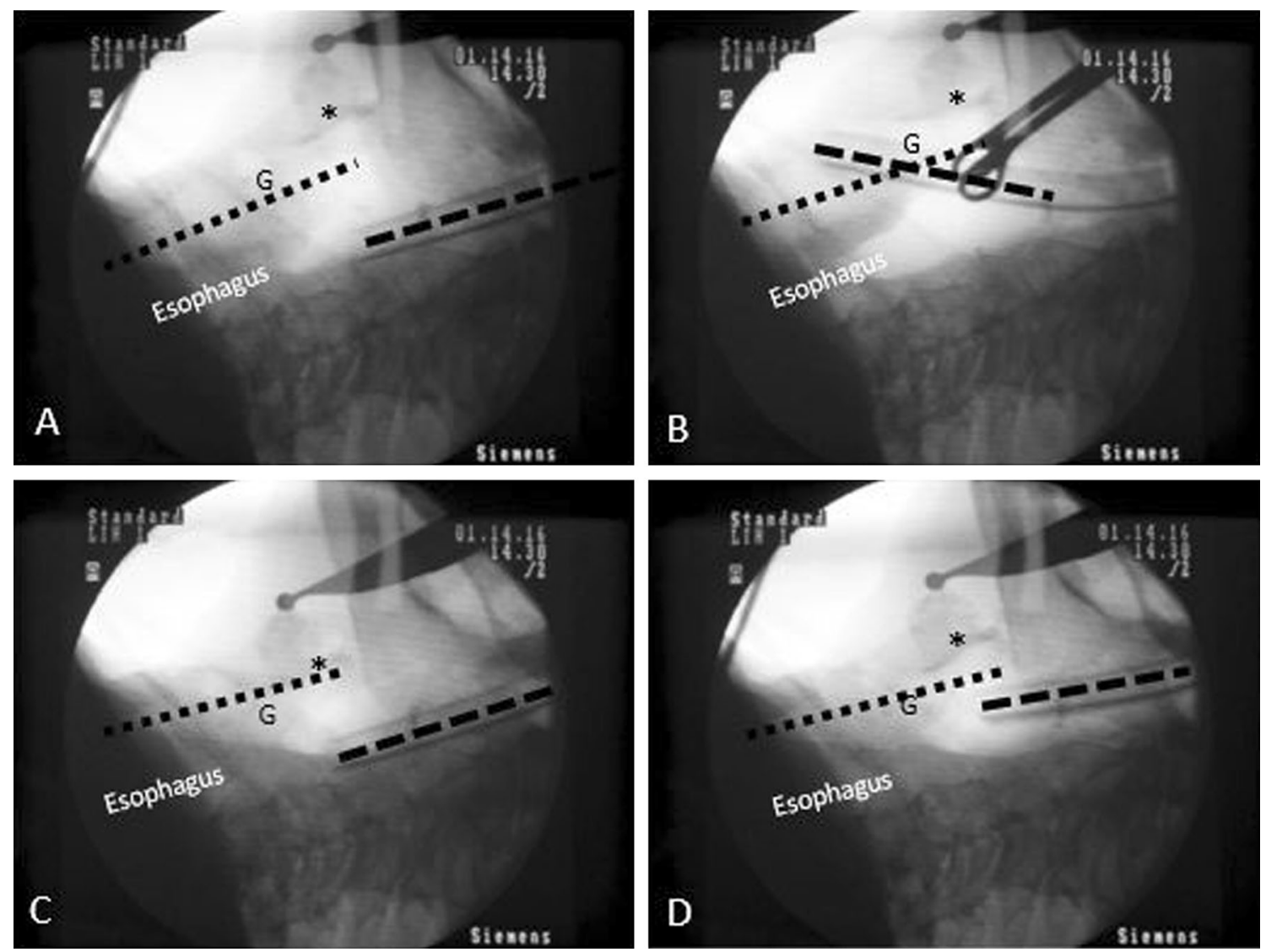

Figure Fluoroscopic view of glottic opening-nasotracheal tube (NTT) alignment during nasotracheal intubation using the C-MAC video laryngoscope. Panel A) Maximal lift for optimal glottic view under direct laryngoscopy using the C-MAC. Note the degree of ventral displacement of the vallecula. Panel B) Maximal lift under direct laryngoscopy using Magill forceps to direct the NTT into the

Using these two techniques - limiting the glottic view with the C-MAC video laryngoscope (Karl Storz Inc., Culver City, CA, USA) and cuff inflation - we successfully performed more than 50 nasotracheal intubations over the last 12 months without requiring Magill forceps. (A video illustrating this technique is available as Electronic Supplementary Material.)

In summary, we encourage airway practitioners to change their approach to nasotracheal intubation by considering glottic opening-NTT alignment their main objective using two simple techniques: limiting the glottic view and cuff inflation.

Conflicts of interest None declared.

glottic opening. Panel C) Minimal lift for a limited view of the glottis under the indirect view using the C-MAC. Panel D) Minimal lift under indirect laryngoscopy with cuff inflation to direct the NTT into the glottic opening. G indicates the glottic opening; * indicates the epiglottis; ..... indicates the tracheal axis; _ _ _ indicates the NTT axis

\section{References}

1. Dorsch JA, Dorsch SE. Tracheal tubes and associated equipment. In: Dorsch JA, Dorsch SE, editors. Understanding Anesthesia Equipment. 5th ed. Philadelphia: Wolters Kluwer Health/Lippincott Williams \& Wilkins; 2008. p. 612.

2. Jones PM, Armstrong KP, Armstrong PM, et al. A comparison of glidescope videolaryngoscopy to direct laryngoscopy for nasotracheal intubation. Anesth Analg 2008; 107: 144-8.

3. Mihai $R$, Blair E, Kay H, Cook TM. A quantitative review and meta-analysis of performance of non-standard laryngoscopes and rigid fibreoptic intubation aids. Anaesthesia 2008; 63: 745-60.

4. Gorback MS. Inflation of the endotracheal tube cuff as an aid to blind nasal endotracheal intubation. Anesth Analg 1987; 66: 916-7.

5. Xue FS, Liu JH, Yuan YJ, Liao X, Wang Q. Cuff inflation as an aid to nasotracheal intubation using the Airtraq laryngoscope. Can J Anesth 2010; 57: 519-20.

Editorial responsibility This submission was handled by Dr. Gregory L. Bryson, Deputy Editor-in-Chief, Canadian Journal of Anesthesia. 